\title{
Two-body Wave Functions, Compositeness, And The Internal Structure Of Dynamically Generated Resonances
}

\section{Takayasu Sekihara*}

Advanced Science Research Center, Japan Atomic Energy Agency, Shirakata, Tokai, Ibaraki, 319-1195, Japan

E-mail: sekiharaepost.j-parc.jp

\section{Tetsuo Hyodo}

Yukawa Institute for Theoretical Physics, Kyoto University, Kyoto 606-8502, Japan

E-mail: hyododyukawa.kyoto-u.ac.jp

\section{Daisuke Jido}

Department of Physics, Tokyo Metropolitan University, Hachioji 192-0397, Japan

E-mail: iidoetmu.ac.jp

\section{Junko Yamagata-Sekihara}

National Institute of Technology, Oshima College, Oshima, Yamaguchi, 742-2193, Japan

E-mail: vamagatadoshima-k.ac.jp

\section{Shigehiro Yasui}

Department of Physics, Tokyo Institute of Technology, Tokyo 152-8551, Japan

E-mail: vasuiseth.phys.titech.ac.jp

\begin{abstract}
Recently, the compositeness, defined as the norm of a two-body wave function for bound and resonance states, has been investigated to discuss the internal structure of hadrons in terms of hadronic molecular components. From the studies of the compositeness, it has been clarified that the two-body wave function of a bound state can be extracted from the residue of the scattering amplitude at the bound state pole. Of special interest is that the two-body wave function from the scattering amplitude is automatically normalized. In particular, while the compositeness is unity for energy-independent interactions, it deviates from unity for energy-dependent interactions, which can be interpreted as a missing-channel contribution. In this manuscript, we show the formulation of the two-body wave function from the scattering amplitude, evaluate the compositeness for several dynamically generated resonances such as $f_{0}(980), \Lambda(1405)$, and $\Xi(1690)$, and investigate their internal structure in terms of the hadronic molecular components.
\end{abstract}

The 26th International Nuclear Physics Conference

11-16 September, 2016

Adelaide, Australia

${ }^{*}$ Speaker. 


\section{Introduction}

Thanks to the recent improvements of the hadron spectroscopy [1], we can discuss not only global quantities of hadrons such as masses and widths but also their internal structure. In particular, from experimental observables we can examine various hadrons for their exotic configurations such as multi-quark states, dibaryons, and hadronic molecules instead of the ordinary configurations, i.e., three quarks for baryons and a quark-antiquark pair for mesons.

In this line, hadronic molecular configuration is of special interest because the structure of hadrons can be expressed in terms of the hadronic degrees of freedom, which are asymptotic states of the fundamental theory of strong interactions, QCD, as distinguishable components [2]. Actually, from decades ago, hadron-hadron scattering amplitudes have been utilized for clarifying the structure of states in terms of hadronic molecules [3,4]. Recently, hadron-hadron scattering amplitudes have been intensively studied so as to investigate the hadronic molecular components for hadronic resonances in the amplitude, and these studies can be characterized by the compositeness [2,5-13], defined as the norm of a two-body wave function interest, based on the fact that the wave function extracted from the scattering amplitude is automatically normalized [14-16].

In the present manuscript, we formulate the two-body wave functions and compositeness from the scattering amplitudes and discuss the internal structure of several dynamically generated hadronic resonances in terms of the hadronic molecular components $[10,12,16]$.

\section{Two-body wave functions and compositeness from scattering amplitudes}

First of all, we consider a quantum system governed by the Hamiltonian $\hat{H}=\hat{H}_{0}+\hat{V}$ with the free part $\hat{H}_{0}$ and interaction $\hat{V}$. We assume that the present model space is restricted to two-body states. The free Hamiltonian $\hat{H}_{0}$ has a two-body eigenstate of its relative momentum $\boldsymbol{q}$ as:

$$
\hat{H}_{0}\left|\boldsymbol{q}_{j}\right\rangle=\mathscr{E}_{j}(q)\left|\boldsymbol{q}_{j}\right\rangle, \quad \mathscr{E}_{j}(q) \equiv \sqrt{q^{2}+m_{j}^{2}}+\sqrt{q^{2}+M_{j}^{2}} \quad \text { (Semi-relativistic case), }
$$

where $q \equiv|\boldsymbol{q}|, j$ is the channel index, and $m_{j}$ and $M_{j}$ are masses of the particles in channel $j$. With this eigenstate, the interaction can be evaluated as $V_{j k}\left(E ; \boldsymbol{q}^{\prime}, \boldsymbol{q}\right)=\left\langle\boldsymbol{q}_{j}^{\prime}|\hat{V}(E)| \boldsymbol{q}_{k}\right\rangle$, where the interaction is allowed to depend intrinsically on the energy of the system $E$.

In this construction, we can consider a two-body to two-body scattering process $k(\boldsymbol{q}) \rightarrow j\left(\boldsymbol{q}^{\prime}\right)$. The scattering amplitude for this process, $T_{j k}\left(E ; \boldsymbol{q}^{\prime}, \boldsymbol{q}\right)$, is a solution of the Lippmann-Schwinger equation, and after the projection to the partial wave of orbital angular momentum $L$ we have

$$
T_{L, j k}\left(E ; q^{\prime}, q\right)=V_{L, j k}\left(E ; q^{\prime}, q\right)+\sum_{l} \int \frac{d k}{2 \pi^{2}} k^{2} \frac{V_{L, j l}\left(E ; q^{\prime}, k\right) T_{L, l k}(E ; k, q)}{E-\mathscr{E}_{l}(k)} .
$$

If the interaction generates a bound state, including an unstable case, the scattering amplitude has a corresponding pole in the complex energy plane. In particular, for an off-shell amplitude, which we may treat as a function of three independent variables $E, q^{\prime}$, and $q$, the pole can be expressed as

$$
T_{L, j k}\left(E ; q^{\prime}, q\right)=\frac{\gamma_{j}\left(q^{\prime}\right) \gamma_{k}(q)}{E-E_{\text {pole }}}+\left(\text { regular at } E=E_{\text {pole }}\right),
$$

with the residue with respect to the pole at $E=E_{\text {pole }}, \gamma_{j}(q)$. 

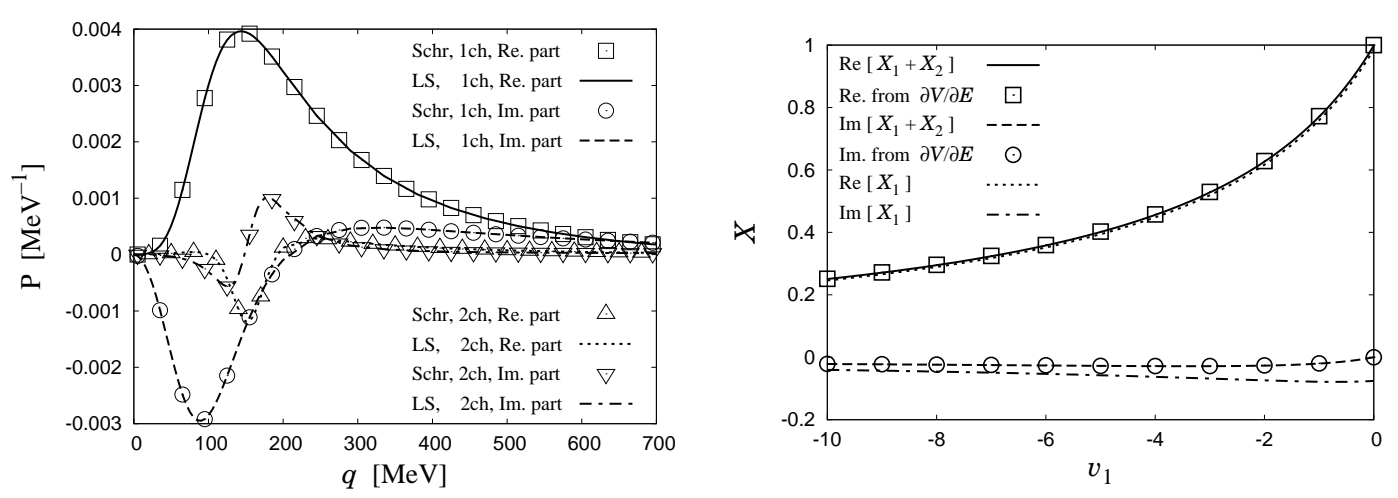

Figure 1: (Left) Density distribution $\mathrm{P}_{j}$ as a function of the momentum $q$ with the interaction ([2.7) [16]. (Right) Compositeness as a function of the parameter $v_{1}$ with the interaction ([2.]) [16].

Interestingly, the residue $\gamma_{j}(q)$ contains information on the wave function of the bound state $|\Psi\rangle$. Actually, in the vicinity of the pole position, we can evaluate the scattering amplitude in the expansion by the eigenstates of the full Hamiltonian, which results in

$$
T_{L, j k}\left(E ; q^{\prime}, q\right) \approx\left\langle\boldsymbol{q}_{j}^{\prime}\left|\hat{V}\left(E_{\text {pole }}\right)\right| \Psi\right\rangle \frac{1}{E-E_{\text {pole }}}\left\langle\tilde{\Psi}\left|\hat{V}\left(E_{\text {pole }}\right)\right| \boldsymbol{q}_{k}\right\rangle,
$$

where we have introduced a Gamow vector $\langle\tilde{\Psi}|$ so as to establish the normalization of the resonance states $[2,14,17]$. Here $\langle\tilde{\Psi} \mid \Psi\rangle=1$ is guaranteed by taking the residue of the propagator $(E-$ $\left.E_{\text {pole }}\right)^{-1}$, which is nothing but the field renormalization constant for the bound state, unity. Then, the residue $\gamma_{j}(q)$ is calculated as

$$
\gamma_{j}(q)=\left\langle\boldsymbol{q}_{j}\left|\hat{V}\left(E_{\mathrm{pole}}\right)\right| \Psi\right\rangle=\left[E_{\mathrm{pole}}-\mathscr{E}_{j}(q)\right]\left\langle\boldsymbol{q}_{j} \mid \Psi\right\rangle,
$$

and similarly for $\left\langle\tilde{\Psi}\left|\hat{V}\left(E_{\text {pole }}\right)\right| \boldsymbol{q}_{j}\right\rangle$. An important property is that the residue $\gamma_{j}(q)$ as well as the scattering amplitude should be automatically normalized because the Lippmann-Schwinger equation is an inhomogeneous integral equation. This fact is especially essential when we calculate the norm of the $j$ th channel component of the wave function, $\left\langle\boldsymbol{q}_{j} \mid \Psi\right\rangle$ :

$$
X_{j} \equiv \int \frac{d^{3} q}{(2 \pi)^{3}}\left\langle\tilde{\Psi} \mid \boldsymbol{q}_{j}\right\rangle\left\langle\boldsymbol{q}_{j} \mid \Psi\right\rangle=\int_{0}^{\infty} d q \mathrm{P}_{j}(q), \quad \mathrm{P}_{j}(q) \equiv \frac{q^{2}}{2 \pi^{2}}\left[\frac{\gamma_{j}(q)}{E_{\mathrm{pole}}-\mathscr{E}_{j}(q)}\right]^{2},
$$

where the norm $X_{j}$ is called compositeness.

The correct normalization of $X_{j}$ from the residue of the scattering amplitude at the pole was proved in Ref. [14] for a general single-channel energy-independent interaction in the nonrelativistic case. For a more general case, one can show that the correct normalization of $X_{j}$ from the amplitude is achieved with some schematic models. For instance, in Fig. W(left) we show the density distribution $\mathrm{P}_{j}(q)$ for a resonance in a two-channel problem where the interaction is

$$
V_{j k}\left(E ; \boldsymbol{q}^{\prime}, \boldsymbol{q}\right)=C_{j k} v(E)(\sqrt{\pi} b)^{3} e^{-\left|\boldsymbol{q}^{\prime}-\boldsymbol{q}\right|^{2} b^{2} / 4}, \quad C_{j k}=\left(\begin{array}{cc}
1 & x \\
x & 0
\end{array}\right), \quad v(E)=v_{0}+v_{1}\left(E-E_{\text {pole }}\right),
$$

with the parameters $b=0.5 \mathrm{fm}, x=0.5, v_{0}=-650 \mathrm{MeV}, v_{1}=0$, and $\left(m_{1}, M_{1}, m_{2}, M_{2}\right)=(495.7$, 938.9, 138.0, 1193.1) MeV. In Fig. W(left), the points are obtained by solving the Schrödinger 
equation and normalizing the wave function $\left(X_{1}+X_{2}=1\right)$ by hand in a usual manner, while the lines by solving the Lippmann-Schwinger equation at the pole position and extracting the wave function from the scattering amplitude. We can see an exact correspondence between the points and lines for each component in Fig. W(left), which means that we obtain the correctly normalized wave function from the scattering amplitude for the energy-independent interaction.

Then, an interesting thing happens when we introduce the energy dependence of the interaction with $v_{1} \neq 0$. Actually, with $v_{1} \neq 0$, the compositeness from the scattering amplitude, which should be automatically normalized, deviates from unity as seen in the lines of Fig. W(right), where we plot the compositeness as a function of $v_{1}$. One interpretation of this behavior is the reflection of a missing-channel contribution. The deviation can be compared with the compositeness in an energy dependent interaction discussed in, e.g., Refs. [18, 19]. According to these studies, the compositeness with the energy-dependent interaction is $[16,18,19]$

$$
X_{\partial V / \partial E}=1+\sum_{j, k} \int_{0}^{\infty} d q \frac{q^{2}}{2 \pi^{2}} \frac{\gamma_{j}(q)}{E_{\text {pole }}-\mathscr{E}_{j}(q)} \int_{0}^{\infty} d q^{\prime} \frac{q^{\prime 2}}{2 \pi^{2}} \frac{\gamma_{k}\left(q^{\prime}\right)}{E_{\text {pole }}-\mathscr{E}_{k}\left(q^{\prime}\right)} \frac{\partial V_{L, j k}}{\partial E}\left(E_{\text {pole }} ; q, q^{\prime}\right),
$$

so that the continuity equation from the wave function should be hold. This compositeness $X_{\partial V / \partial E}$ is shown in Fig. W(right) as points, which lies exactly on the lines of the real and imaginary parts of $X_{1}+X_{2}$. This indicates that the two-body wave function from the scattering amplitude correctly takes into account the effect of the additional second term in (2.8) due to the energy dependence of the interaction.

\section{Compositeness for dynamically generated hadronic resonances}

Next, we evaluate the compositeness for several dynamically generated hadronic resonances, which are described not in quark-gluon but in hadronic degrees of freedom and hence may be hadronic molecules.

Before calculating the compositeness for hadrons, we mention that, because the compositeness as well as the wave function itself is not a physical observable, the compositeness is a model dependent quantity [9]. An exception is the case that the pole exists very close to the on-shell energies, where we can model-independently express the compositeness with the scattering length and effective range $[3,4,7,11]$. Besides, as described above, the compositeness from the scattering amplitude is uniquely determined once we fix the model space and interaction.

In the present study we fix the interaction as the separable form for the orbital angular momentum $L$ [6]

$$
\left\langle\boldsymbol{q}_{j}^{\prime}|\hat{V}(E)| \boldsymbol{q}_{k}\right\rangle=(2 L+1) q^{L} q^{L} P_{L}\left(\hat{q}^{\prime} \cdot \hat{q}\right) V_{j k}(E),
$$

where $\hat{q}^{(\prime)} \equiv \boldsymbol{q}^{(\prime)} /\left|\boldsymbol{q}^{(\prime)}\right|$. Then, the Lippmann-Schwinger equation (2.2) becomes an algebraic form:

$$
T_{j k}(E)=V_{j k}(E)+\sum_{l} V_{j l}(E) G_{l}(E) T_{l k}(E), \quad G_{j}(E) \equiv \int \frac{d^{3} k}{(2 \pi)^{3}} \frac{k^{2 L}}{E-\mathscr{E}_{j}(k)},
$$

where the ultraviolet divergence in the loop function $G_{j}(E)$ is regularized in a certain way. Then, the residue $\gamma_{j}$ becomes a coupling constant and hence the compositeness is $[10,12]$

$$
X_{j}=-\gamma_{j}^{2}\left[\frac{d G_{j}}{d E}\right]_{E=E_{\mathrm{pole}}} .
$$


Table 1: Compositeness for dynamically generated hadronic resonances: scalar mesons.

\begin{tabular}{lcccc}
\hline \hline & $f_{0}(500)[10]$ & $f_{0}(980)[10]$ & $a_{0}(980)[23]$ & $K_{0}^{*}(800)[10]$ \\
\hline$E_{\text {pole }}[\mathrm{MeV}]$ & $443-217 i$ & $988-4 i$ & $979-53 i$ & $750-227 i$ \\
$X_{\pi \pi}$ & $-0.09+0.37 i$ & $0.00-0.00 i$ & - & - \\
$X_{\pi \eta}$ & - & - & $-0.06+0.10 i$ & - \\
$X_{K \bar{K}}$ & $-0.01-0.00 i$ & $0.87-0.04 i$ & $0.38-0.29 i$ & - \\
$X_{\eta \eta}$ & $-0.00+0.00 i$ & $0.06+0.01 i$ & - & - \\
$X_{\pi K}$ & - & - & - & $0.32+0.36 i$ \\
$X_{\eta K}$ & - & - & - & $-0.01-0.00 i$ \\
$Z$ & $1.09-0.37 i$ & $0.07+0.02 i$ & $0.68+0.18 i$ & $0.70-0.36 i$ \\
$U$ & 0.54 & 0.00 & 0.30 & 0.28 \\
$\tilde{X}_{\pi \pi}$ & 0.25 & 0.00 & - & - \\
$\tilde{X}_{\pi \eta}$ & - & - & 0.09 & - \\
$\tilde{X}_{K \bar{K}}$ & 0.01 & 0.87 & 0.37 & - \\
$\tilde{X}_{\eta \eta}$ & 0.00 & 0.06 & - & - \\
$\tilde{X}_{\pi K}$ & - & - & - & 0.38 \\
$\tilde{X}_{\eta K}$ & - & - & - & 0.01 \\
$\tilde{Z}$ & 0.75 & 0.07 & 0.54 & 0.62 \\
\hline \hline
\end{tabular}

In addition, we can define the missing-channel contribution $Z$ as the rest part for the normalization of the total wave function, i.e., unity minus the sum of the compositeness:

$$
Z \equiv 1-\sum_{j} X_{j}=-\sum_{j, k} \gamma_{k} \gamma_{j}\left[G_{j} \frac{d V_{j k}}{d E} G_{k}\right]_{E=E_{\mathrm{pole}}},
$$

where the sum rule for $X_{j}$ and $Z$ was proved in Ref. [20]. We also note that the compositeness and missing-channel contribution are in general complex for resonances, which cannot be interpreted as probabilities. Here, from the complex $X_{j}$ and $Z$ we introduce quantities which a probabilistic interpretation is possible:

$$
\tilde{X} \equiv \frac{1+|X|-|Z|}{2}, \quad \tilde{Z} \equiv \frac{1+|Z|-|X|}{2}
$$

for the one-channel problem, and

$$
\tilde{X}_{j} \equiv \frac{\left|X_{j}\right|}{1+U}, \quad \tilde{Z} \equiv \frac{|Z|}{1+U}, \quad U \equiv \sum_{j}\left|X_{j}\right|+|Z|-1,
$$

for the problems with more than one channel. In any case, based on a similarity between the resonance wave function considered and a wave function of a stable bound state, with $U \ll 1$ one can safely interpret $\tilde{X}_{j}(\tilde{Z})$ as the probability of finding the composite (missing) part.

Now we calculate the compositeness for hadronic resonances. In this study we employ chiral perturbation theory for the separable interaction (B.D), with which we can dynamically generate several hadronic resonances. Here we treat $f_{0}(500), f_{0}(980), a_{0}(980)$, and $K_{0}^{*}(800)$ for mesons and $\Delta(1232), N(1535), N(1650), \Lambda(1405), \Lambda(1670)$, and $\Xi(1690)$ for baryons. In particular, the scattering amplitudes for the scalar mesons $f_{0}(500), f_{0}(980)$, and $K_{0}^{*}(800)$ are taken from Ref. [21], and for $\Lambda(1405)$ from Ref. [22]. The results are listed in Tables [, 口, and B]. 
Table 2: Compositeness for dynamically generated hadronic resonances: baryons with $S=0$.

\begin{tabular}{lccc}
\hline \hline & $\Delta(1232)^{1}[12]$ & $N(1535)[12]$ & $N(1650)[12]$ \\
\hline$E_{\text {pole }}[\mathrm{MeV}]$ & $1207-50 i$ & $1496-59 i$ & $1661-70 i$ \\
$X_{\pi N}$ & $0.87+0.35 i$ & $-0.02+0.03 i$ & $0.00+0.04 i$ \\
$X_{\eta N}$ & - & $0.04+0.37 i$ & $0.00+0.01 i$ \\
$X_{K \Lambda}$ & - & $0.14+0.00 i$ & $0.08+0.05 i$ \\
$X_{K \Sigma}$ & - & $0.01-0.02 i$ & $0.09-0.12 i$ \\
$Z$ & $0.13-0.35 i$ & $0.84-0.38 i$ & $0.84+0.01 i$ \\
$U$ & 0.31 & 0.48 & 0.13 \\
$\tilde{X}_{\pi N}$ & 0.78 & 0.03 & 0.04 \\
$\tilde{X}_{\eta N}$ & - & 0.25 & 0.01 \\
$\tilde{X}_{K \Lambda}$ & - & 0.09 & 0.08 \\
$\tilde{X}_{K \Sigma}$ & - & 0.01 & 0.13 \\
$\tilde{Z}$ & 0.22 & 0.62 & 0.74 \\
\hline \hline
\end{tabular}

First, among the scalar mesons in Table 田, the $K \bar{K}$ compositeness for the $f_{0}(980)$ resonance, both $X_{K \bar{K}}$ and $\tilde{X}_{K \bar{K}}$, is close to unity with negligible value of $U$. This result suggests that $f_{0}(980)$ in this model is indeed a $K \bar{K}$ molecular state. On the other hand, the other scalar mesons have large contributions from the missing channels, $Z$. Among them, however, $f_{0}(500)$ has the value of $U$ comparable to unity, so one cannot clearly interpret the structure of $f_{0}(500)$ from $X$ and $Z$.

Next, from the results for the nucleon resonances in Table $\square$, one can see that the $\Delta(1232)$ resonance has a large contribution from the $\pi N$ component, as in the previous work [8]. The imaginary part of the $\pi N$ compositeness for $\Delta(1232)$ is also non-negligible, but $U$ is less than onethird, so we may conclude that the $\pi N$ component in $\Delta(1232)$ is significant. On the other hand, the results on $N(1535)$ and $N(1650)$ indicate that the $\pi N, \eta N, K \Lambda$, and $K \Sigma$ components are negligible for these resonances.

The results for the baryons with $S \leq-1$ are shown in Table B]. An interesting finding is that the higher pole of $\Lambda(1405)$ has a dominant $\bar{K} N$ compositeness $X_{\bar{K} N}$, which is very close to unity. Although the value of $U$ is non-negligible compared to unity for the higher $\Lambda(1405)$, this mainly comes from the imaginary parts and negative real parts of contributions other than $\bar{K} N$, and $U$ divided by the number of channels is small: $U / 5 \approx 0.15 \ll 1$. Therefore, we may state that the higher $\Lambda(1405)$ is dominated by the $\bar{K} N$ composite component. The $\Lambda(1670)$ resonance has substantial $K \Xi$ component in the present model. Finally, the result on $\Xi(1690)$ indicates that this resonance is indeed a $\bar{K} \Sigma$ molecular state.

\section{Summary}

We have shown that the two-body wave function of a bound/resonance state can be evaluated from the scattering amplitudes as an automatically normalized quantity. In particular, the compositeness, i.e., the norm of the two-body wave function, is unity for an energy-independent interaction, while the compositeness deviates from unity for an energy-dependent interaction, which can be interpreted to implement a missing-channel contribution.

\footnotetext{
${ }^{1}$ We only consider the $\pi N$ channel for the $\Delta(1232)$ resonance.
} 
Table 3: Compositeness for dynamically generated hadronic resonances: baryons with $S \leq-1$.

\begin{tabular}{|c|c|c|c|c|}
\hline & $\Lambda(1405)_{\text {higher }}[10]$ & $\Lambda(1405)_{\text {lower }}[10]$ & $\Lambda(1670)[10]$ & $\Xi(1690)[24]$ \\
\hline$E_{\text {pole }}[\mathrm{MeV}]$ & $1424-26 i$ & $1381-81 i$ & $1678-21 i$ & $1684-1 i$ \\
\hline$X_{\bar{K} N}$ & $1.14+0.01 i$ & $-0.39-0.07 i$ & $0.03+0.00 i$ & - \\
\hline$X_{\pi \Sigma}$ & $-0.19-0.22 i$ & $0.66+0.52 i$ & $0.00+0.00 i$ & - \\
\hline$X_{\eta \Lambda}$ & $0.13+0.02 i$ & $-0.04+0.01 i$ & $-0.09+0.16 i$ & - \\
\hline$X_{K \Xi}$ & $0.00+0.00 i$ & $-0.00+0.00 i$ & $0.53-0.10 i$ & - \\
\hline$X_{\bar{K} \Sigma}$ & - & - & - & $0.95-0.14 i$ \\
\hline$X_{\bar{K} \Lambda}$ & - & - & - & $-0.02+0.00 i$ \\
\hline$X_{\pi \Xi}$ & - & - & - & $0.00+0.00 i$ \\
\hline$X_{\eta \Xi}$ & - & - & - & $0.01+0.02 i$ \\
\hline$Z$ & $-0.08+0.19 i$ & $0.77-0.46 i$ & $0.53-0.06 i$ & $0.06+0.11 i$ \\
\hline$U$ & 0.77 & 1.17 & 0.29 & 0.13 \\
\hline$\tilde{X}_{\bar{K} N}$ & 0.64 & 0.18 & 0.02 & - \\
\hline$\tilde{X}_{\pi \Sigma}^{\Lambda v}$ & 0.16 & 0.39 & 0.00 & - \\
\hline$\tilde{X}_{\eta \Lambda}$ & 0.07 & 0.02 & 0.14 & - \\
\hline$\tilde{X}_{K \Xi}^{\prime}$ & 0.00 & 0.00 & 0.42 & - \\
\hline$\tilde{X}_{\bar{K} \Sigma}$ & - & - & - & 0.85 \\
\hline$\tilde{X}_{\bar{K} \Lambda}^{K L}$ & - & - & - & 0.02 \\
\hline$\tilde{X}_{\pi \Xi}$ & - & - & - & 0.00 \\
\hline$\tilde{X}_{\eta \Xi}$ & - & - & - & 0.02 \\
\hline$\tilde{Z}$ & 0.12 & 0.41 & 0.41 & 0.11 \\
\hline
\end{tabular}

By evaluating the compositeness for several dynamically generated resonances with the interaction taken from chiral perturbation theory, we conclude that $f_{0}(980), \Lambda(1405)$, and $\Xi(1690)$ are dominated by the $K \bar{K}, \bar{K} N$, and $\bar{K} \Sigma$ composite components, respectively, in the present model.

\section{References}

[1] C. Patrignani et al. [Particle Data Group], The Review of Particle Physics, Chin. Phys. C 40 (2016) 100001.

[2] T. Hyodo, Structure and compositeness of hadron resonances, Int. J. Mod. Phys. A 28 (2013) 1330045 [arXiv:1310.1176 [hep-ph]].

[3] S. Weinberg, Evidence That the Deuteron Is Not an Elementary Particle, Phys. Rev. 137 (1965) B672.

[4] V. Baru, J. Haidenbauer, C. Hanhart, Y. Kalashnikova and A. E. Kudryavtsev, Evidence that the $a_{0}(980)$ and $f_{0}(980)$ are not elementary particles, Phys. Lett. B 586 (2004) 53 [hep-ph/0308129].

[5] T. Hyodo, D. Jido and A. Hosaka, Compositeness of dynamically generated states in a chiral unitary approach, Phys. Rev. C 85 (2012) 015201 [arXiv:1108.5524 [nucl-th]].

[6] F. Aceti and E. Oset, Wave functions of composite hadron states and relationship to couplings of scattering amplitudes for general partial waves, Phys. Rev. D 86 (2012) 014012 [arXiv:1202.4607 [hep-ph]].

[7] T. Hyodo, Structure of Near-Threshold s-Wave Resonances, Phys. Rev. Lett. 111 (2013) 132002 [arXiv:1305.1999 [hep-ph]]. 
[8] F. Aceti, L. R. Dai, L. S. Geng, E. Oset and Y. Zhang, Meson-baryon components in the states of the baryon decuplet, Eur. Phys. J. A 50 (2014) 57 [arXiv:1301.2554 [hep-ph]].

[9] H. Nagahiro and A. Hosaka, Elementarity of composite systems, Phys. Rev. C 90 (2014) 065201 [arXiv:1406.3684 [hep-ph]].

[10] T. Sekihara, T. Hyodo and D. Jido, Comprehensive analysis of the wave function of a hadronic resonance and its compositeness, PTEP 2015 (2015) 063D04 [arXiv:1411.2308 [hep-ph]].

[11] Y. Kamiya and T. Hyodo, Structure of near-threshold quasibound states, Phys. Rev. C 93 (2016) 035203 [arXiv:1509.00146 [hep-ph]]; Generalized weak-binding relations of compositeness in effective field theory, PTEP 2017 (2017), in press [arXiv:1607.01899 [hep-ph]].

[12] T. Sekihara, T. Arai, J. Yamagata-Sekihara and S. Yasui, Compositeness of baryonic resonances: Application to the $\Delta(1232), N(1535)$, and N(1650) resonances, Phys. Rev. C 93 (2016) 035204 [arXiv:1511.01200 [hep-ph]].

[13] Z. H. Guo and J. A. Oller, Probabilistic interpretation of compositeness relation for resonances, Phys. Rev. D 93 (2016) 096001 [arXiv:1508.06400 [hep-ph]].

[14] E. Hernandez and A. Mondragon, Resonant states in momentum representation, Phys. Rev. C 29 (1984) 722.

[15] D. Gamermann, J. Nieves, E. Oset and E. Ruiz Arriola, Couplings in coupled channels versus wave functions: Application to the X(3872) resonance, Phys. Rev. D 81 (2010) 014029 [arXiv:0911.4407 [hep-ph]]; J. Yamagata-Sekihara, J. Nieves and E. Oset, Couplings in coupled channels versus wave functions in the case of resonances: Application to the two $\Lambda(1405)$ states, Phys. Rev. D 83 (2011) 014003 [arXiv:1007.3923 [hep-ph]].

[16] T. Sekihara, Two-body wave functions and compositeness from scattering amplitudes: General properties with schematic models, arXiv:1609.09496 [quant-ph].

[17] G. Gamow, Zur Quantentheorie des Atomkernes, Z. Phys. 51 (1928) 204.

[18] J. Formánek, R. J. Lombard, J. Mareš, Wave equations with energy-dependent potentials, Czech. J. Phys. 54 (2004) 289 [arXiv:0309157 [quant-ph]].

[19] K. Miyahara and T. Hyodo, Structure of $\Lambda(1405)$ and construction of $\bar{K} N$ local potential based on chiral SU(3) dynamics, Phys. Rev. C 93 (2016) 015201 [arXiv:1506.05724 [nucl-th]].

[20] T. Sekihara, T. Hyodo and D. Jido, Internal structure of the resonant $\Lambda(1405)$ state in chiral dynamics, Phys. Rev. C 83 (2011) 055202 [arXiv:1012.3232 [nucl-th]].

[21] A. Gomez Nicola and J. R. Pelaez, Meson-meson scattering within one-loop chiral perturbation theory and its unitarization, Phys. Rev. D 65 (2002) 054009 [hep-ph/0109056].

[22] Y. Ikeda, T. Hyodo and W. Weise, Improved constraints on chiral SU(3) dynamics from kaonic hydrogen, Phys. Lett. B 706 (2011) 63 [arXiv:1109.3005 [nucl-th]]; Chiral SU(3) theory of antikaon-nucleon interactions with improved threshold constraints, Nucl. Phys. A 881 (2012) 98 [arXiv:1201.6549 [nucl-th]].

[23] T. Sekihara and T. Hyodo, Size measurement of dynamically generated hadronic resonances with finite boxes, Phys. Rev. C 87 (2013) 045202 [arXiv:1209.0577 [nucl-th]].

[24] T. Sekihara, $\Xi(1690)$ as a $\bar{K} \Sigma$ molecular state, PTEP 2015 (2015) $091 \mathrm{D} 01$ [arXiv:1505.02849 [hep-ph]]. 